The majority of EDs in England have now adopted GPED. The increase in Inside/parallel models and the reduction in Inside/integrated models is likely to be related to the availability of capital funding to finance structural changes to EDs so that separate GP services could be provided. Further research is required to understand the relative effectiveness of the various models of GPED identified.

\section{THE END-TIDAL AND ARTERIAL CARBON DIOXIDE GRADIENT IN SERIOUS TRAUMATIC BRAIN INJURY AFTER PRE-HOSPITAL EMERGENCY ANAESTHESIA: A RETROSPECTIVE OBSERVATIONAL STUDY}

${ }^{1}$ James Price, ${ }^{2}$ Daniel Sandbach, ${ }^{1}$ Ari Ercole, ${ }^{2}$ Alastair Wilson, ${ }^{2}$ Ed Barnard. ${ }^{1}$ Cambridge University Hospitals NHS Foundation Trust; ${ }^{2}$ East Anglian Air Ambulance

\subsection{6/emj-2020-rcemabstracts.43}

Aims/Objectives/Background In the United Kingdom (UK), 20\% of patients with severe traumatic brain injury (TBI) receive prehospital emergency anaesthesia (PHEA). Current guidance recommends an end-tidal carbon dioxide $\left(\mathrm{ETCO}_{2}\right)$ of $4.0-4.5 \mathrm{kPa}$ to achieve a low-normal arterial partial pressure of $\mathrm{CO}_{2}\left(\mathrm{PaCO}_{2}\right)$, and reduce secondary brain injury. This recommendation assumes a $0.5 \mathrm{kPa} \mathrm{ETCO}_{2}-\mathrm{PaCO}_{2}$ gradient. However, the gradient in the acute phase of TBI is unknown. Our primary aim was to report the $\mathrm{ETCO}_{2}-\mathrm{PaCO}_{2}$ gradient of TBI patients at hospital arrival.

Methods/Design A retrospective cohort study of adult patients with serious TBI, who received a PHEA by a pre-hospital critical care team in the East of England between 1st April 2015 to 31st December 2017. Linear regression was performed to test for correlation and reported as R-squared $\left(\mathrm{R}^{2}\right)$. A BlandAltman plot was used to test for paired $\mathrm{ETCO}_{2}$ and $\mathrm{PaCO}_{2}$ agreement and reported with 95\% confidence intervals $(95 \%$ $\mathrm{CI})$. $\mathrm{ETCO}_{2}-\mathrm{PaCO}_{2}$ gradient data were compared with a twotailed, unpaired, t-test.

Results/Conclusions 107 patients were eligible for inclusion. Sixty-seven patients did not receive a $\mathrm{PaCO}_{2}$ sample within 30 minutes of hospital arrival and were therefore excluded. Forty patients had complete data and were included in the final analysis; per protocol.

The mean $\mathrm{ETCO}_{2}-\mathrm{PaCO}_{2}$ gradient was $1.7( \pm 1.0) \mathrm{kPa}$, with only moderate correlation of $\mathrm{ETCO}_{2}$ and $\mathrm{PaCO}_{2}$ at hospital arrival $\left(\mathrm{R}^{2}=0.23, p=0.002\right)$. The Bland-Altman bias was $1.7(95 \% \mathrm{CI} 1.4-2.0) \mathrm{kPa}$ with upper and lower limits of agreement of $3.6(95 \% \mathrm{CI} 3.0-4.1) \mathrm{kPa}$ and -0.2 (95\%CI $-0.8-$ $0.3) \mathrm{kPa}$ respectively. There was no significant gradient correlation in patients with a co-existing serious thoracic injury $\left(\mathrm{R}^{2}=0.13, p=0.10\right)$, and this cohort had a larger $\mathrm{ETCO}_{2^{-}}$ $\mathrm{PaCO}_{2}$ gradient, $2.0( \pm 1.1) \mathrm{kPa}, p=0.01$. Patients who underwent pre-hospital arterial blood sampling had an arrival $\mathrm{PaCO}_{2}$ of $4.7( \pm 0.2) \mathrm{kPa}$.

Lower $\mathrm{ETCO}_{2}$ targets than previously recommended may be safe and appropriate. The use of pre-hospital $\mathrm{PaCO}_{2}$ measurement is advocated.

\section{CURRENT MANAGEMENT OF MODERATE/SEVERE TRAUMATIC PNEUMOTHORACES: A SURVEY OF EMERGENCY CLINICIANS}

${ }^{1}$ Edward Carlton, ${ }^{2}$ Millie Watkins, ${ }^{1}$ Pascale Avery. ${ }^{1}$ North Bristol NHS Trust; ${ }^{2}$ University of Bristol Medical School

10.1136/emj-2020-rcemabstracts.44
Aims/Objectives/Background Traumatic pneumothoraces are present in one-fifth of multiple trauma victims. Traditional teaching mandates the insertion of a chest drain in the majority of cases. However, recent retrospective observational evidence suggests a trend towards conservative management. The aim of this survey was to understand the current emergency medicine (EM) practice in placing chest drains for the management of moderate/severe traumatic pneumothoraces.

Methods/Design This scoping survey was developed through expert consensus. To achieve face validity, clinical sensibility testing was performed using a pilot survey (with user feedback). There were 14 pilot-phase respondents. The survey was then modified to achieve content validity. The survey was sent electronically to senior EM doctors in 15 sites internationally, and involved six clinical/imaging vignettes asking how likely are you to insert an intercostal chest drain to manage the pneumothorax in ED?'. A five-point response was available from very unlikely to very likely. All pneumothroraces were $>1 \mathrm{~cm}$ on imaging, but mechanism, physiology and need for ventilation varied.

Results/Conclusions Of a potential 409 respondents, 141 responses were received (34\% response rate). Respondents had a range of clinical experience, with the majority qualified more than 10 years (median; 19 years).

For $5 / 6$ cases chest drain insertion was likely or very likely in $>50 \%$ of responses, ranging from $52 \%$ for a non-compromised $1 \mathrm{~cm}$ pneumothorax in a ventilated patient to $98 \%$ for a tension pneumothorax on chest $\mathrm{x}$-ray. Chest drain insertion was unlikely or very unlikely (62\% of responses) in one case; an 86-year-old female on rivaroxaban with moderate respiratory compromise (respiratory rate $30 \mathrm{~min}^{-1}$ ) and a $2 \mathrm{~cm}$ pneumothorax.

There is a broad range of clinical practice involving both conservative and invasive strategies in the treatment of moderate/severe traumatic pneumothoraces. There is clinical equipoise for interventional trials to determine the optimal management strategy for this patient group.

\section{FINDING VOICES: YOUNG PEOPLE'S EXPERIENCES OF THE EMERGENCY DEPARTMENT}

${ }^{1}$ Liza Keating, ${ }^{2}$ Sarah Wilson, ${ }^{3}$ Jonathan Hill, ${ }^{4}$ Jack Dainty. ${ }^{1}$ Royal Berkshire NHS Foundation Trust; ${ }^{2}$ Frimley Health NHS Foundation Trust; ${ }^{3}$ University of Reading; ${ }^{4}$ University of East Anglia

\subsection{6/emj-2020-rcemabstracts.45}

Aims/Objectives/Background Self-harm among adolescents is a major concern both because it gives rise to considerable distress and disruption in young people's lives and it commonly recurs. There are currently wide variations in the care of this group of patients and it is widely reported that their experience in the emergency department is poor. Young people who have self-harmed may differ from others attending the ED and we need to know more about these differences to inform ED care and potential improvements. The aim of this study was to establish the needs and expectations among children and young people in the ED and to increase the understanding of the specific needs of adolescents who self-harm through comparison with another patient group in the ED. 
Methods/Design We undertook a case-control study with adolescents attending for suspected fractures serving as the control group. Adolescents and their guardians were each given a questionnaire pack on arrival in $\mathrm{ED}$, and again at least 2 hours later, thus capturing their expectations and pre-existing characteristics, and their experience. Trained research assistants were present in the ED seven days a week and covered 10 am till $10 \mathrm{pm}$. The study commenced in July 2019 and terminated early in March 2020 at the onset of the COVID 19 pandemic.

Results/Conclusions Young people who had self-harmed had significantly higher mean dissatisfaction scores than those with suspected fractures. They also had higher mean levels of emotional and interpersonal difficulties and these were associated with higher dissatisfaction scores. This is the first case-control study to show that dissatisfaction with the ED is at least partly a function of the particular mental health problems suffered by adolescents who self-harm. This in turn provides initial clues to the particular needs of this group of patients in whom the current management is widely reported as inadequate.

\section{ACCESS TO EMERGENCY HEALTH CARE IN TIGER RESERVES IN THE CENTRAL INDIAN LANDSCAPE. LESSONS FOR GLOBAL EMERGENCY CARE}

${ }^{1}$ Chet Trivedy, ${ }^{2}$ Vijaykumar Tate, ${ }^{2}$ Sheetal Kolhe, ${ }^{2}$ Vishal Gadre, ${ }^{2}$ Anish Andheria. ${ }^{1}$ Brighton and Sussex University Hospital; ${ }^{2}$ Wildlife Conservation Trust (Mumbai)

10.1136/emj-2020-rcemabstracts.46

Aims/Objectives/Background Forest staff working in remote locations are at high risk of life-threatening emergencies; including major trauma and snakebites. Timely access to appropriate emergency care is pivotal to life-saving treatment. This is the first study to systematically map public health facilities (PHFs) around tiger reserves (TRs) in central Indian state of Maharashtra.

Methods/Design Using publically available data; GPS coordinates, medical staff, and services were identified for PHFs in increasing order of specialism; including Primary Healthcare Centre (PHC), Community Health Centre (CHC), Sub-district Hospital (SDH) and District Hospital (DH). GIS Network Analysis was used to identify the nearest PHFs in relation to the access points of eight TRs; and the shortest distance by road and corresponding travel time by vehicle were calculated.

\begin{tabular}{|c|c|c|}
\hline $\begin{array}{l}\text { Type of } \\
\text { Health Facility } \\
(n=96)\end{array}$ & $\begin{array}{l}\text { Average distance from a TR access } \\
\text { point to the nearest PHF in } \\
\text { Kilometers }\end{array}$ & $\begin{array}{l}\text { Average time to reach the } \\
\text { nearest PHF in minutes by a } \\
\text { car }\end{array}$ \\
\hline PHC (38.5\%) & $12.3 \pm 8.6(0.7-39.4)$ & $24 \pm 17(1-79)$ \\
\hline CHC (26\%) & $24.9 \pm 12.4(2.5-61.6)$ & $47 \pm 25(5-123)$ \\
\hline SDH $(13.5 \%)$ & $42 \pm 15.8(4.7-80.9)$ & $82 \pm 32(8-162)$ \\
\hline DH $(22 \%)$ & $65.4 \pm 28.9(11.7-137.2)$ & $126 \pm 57(23-235)$ \\
\hline
\end{tabular}

Results/Conclusions Of all PHFs, $87.5 \%$ offered basic emergency care, while only $54 \%$ offered radiology services. Of all trauma beds $99.9 \%$ were placed at $\mathrm{DH}$ level; along with $89 \%$ of all MBBS trained doctors. Only $28.6 \%$ of TR exit points had access to emergency ambulance service based within 30 minutes.

Conclusion This study highlight the challenges of providing emergency healthcare in low- and middle-income countries and the urgent need for greater resources and infrastructure to support the delivery of emergency care for frontline forest staff in rural areas. Further work in progress to look at the provision of emergency care in remote settings.

\section{THE PROGNOSTIC VALUE OF D-DIMER FOR COVID-19 OUTCOMES}

Peter Killeen, Chet Trivedy, Dave Paradise, Evan Coughlan. Brighton and Sussex University Hospitals NHS Trust

\subsection{6/emj-2020-rcemabstracts.47}

Aims/Objectives/Background The diagnosis and management of COVID-19 has presented a novel challenge in the emergency department (ED). Early and sensitive predictors of outcome are needed to improve management of COVID-19 patients. Recent evidence has suggested a role for a COVID-19 associated pro-thrombotic coagulopathy as part of the underlying pathology. The aim was to evaluate the prognostic utility of D-dimer as a biomarker predictive of outcome in COVID-19 patients.

Methods/Design We retrospectively analysed data for 326 cases of confirmed COVID-19 presenting to our ED at the Royal Sussex County Hospital in Brighton between 13th March and 17th June 2020. During this period 2687 attendances were triaged to the 'red' COVID-19 zone with symptoms suspicious for COVID-19, amongst whom 326 admissions were confirmed to have COVID-19 by CT, chest x-radiograph or PCR swab. D-dimers were measured in ED for 265/326. Peak Ddimer measured during admission was collected to evaluate deteriorations subsequent to admission. Receiver-operating characteristic curves were used to determine an optimal cutoff for discrimination.

Results/Conclusions D-Dimer elevation $>0.5 \mu \mathrm{g} / \mathrm{mL}$ was seen in $93.5 \%$ of admitted patients with confirmed COVID-19. Multivariable logistic regression suggested that age $>75$ $(\mathrm{OR}=3.01 \quad 95 \% \quad \mathrm{CI} \quad 1.65-5.49 \mathrm{p}=0.0003)$ and $\mathrm{D}$-dimer (measured in ED) $>1.25 \mu \mathrm{g} / \mathrm{mL}(\mathrm{OR}=2.0695 \%$ CI $1.08-3.93$ $\mathrm{p}=0.0276$ ) were associated with increased mortality. D-dimer measured in ED predicted mortality with sensitivity of $76.5 \%$ and specificity of $41.3 \%$. The $\mathrm{D}$-dimer rose by $>1.00$ for $30 / 265$ patients subsequent to admission of whom $8 / 30$ (26.7\%) died (all mortality 16\%) and 11/30 (36.7\%) were escalated to intensive care. Peak D-dimer measured during admission $>3.2 \mu \mathrm{g} / \mathrm{mL}$ predicted hospital mortality with $50 \%$ sensitivity and $72.4 \%$ specificity $(\mathrm{OR}=2.16 \quad 95 \%$ CI $1.16-$ $3.99 \mathrm{p}=0.0145)$.

The results of this study support the growing argument that a raised D-dimer may play an important role as a prognostic marker in patients with COVID-19, perhaps indicative of a pro-thrombotic coagulopathy within the underlying pathology. 